\title{
Referral Rates Vary Widely Between Family Medicine Practices
}

\author{
Hamza El Ayadi, BS, Alpa Desai, DO, Ross E. Jones, MD, Elvira Mercado, MD, \\ Maribeth Williams, MD, MSCR, Benjamin Rooks, MS, and Peter J. Carek, MD, MS
}

Introduction: Referral rates and patterns to specialists by family physicians have a significant impact on numerous aspects of medical care, including the quality and cost of care provided. The aim of this study was to examine the referral rate and pattern of family physician practices associated with a large academic health center.

Methods: We conducted a retrospective chart review of referrals for patients seen in the family medicine clinics associated with a large academic institution. Basic demographic information was collected. The primary outcome was the monthly referral rate, measured as the proportion of patient visits that resulted in a consult to a specialist outside of the referring family medicine clinic. Binomial and $\gamma$ generalized linear mixed models were fit to determine the associations between high/medium/low referral rate clinics and patient demographics.

Results: The average monthly referral rate for all of the clinics was $20.3 \%$, and the rate varied significantly between clinic groups. Patient gender, race, and being in a committed relationship were noted to be associated with lower referral rate.

Discussion: Referral rates among family physician practices differ widely and are associated with gender, race, and relationship status of patients. Referrals to several specific specialties are common among family physicians. (J Am Board Fam Med 2021;34:1183-1188.)

Keywords: Family Medicine, Family Physicians, Managed Care Programs, Referral and Consultation, Retrospective Studies

Referral rates and patterns to specialists by family physicians have a significant impact on numerous aspects of medical care, including the quality and cost of care provided. ${ }^{1-7}$ Importantly, the appropriate use of referrals decreases diagnostic uncertainty and assists with the institution of appropriate treatment, resulting in improved quality of care measures. $^{1-4}$ Alternatively, the inappropriate use of referrals can be costly and inefficient, resulting in additional and potentially unnecessary tests and

This article was externally peer reviewed.

Submitted 20 May 2021; revised 19 August 2021; accepted 19 August 2021.

From Department of Community Health and Family Medicine, University of Florida (HEA, AD, EM, MW, BR, PJC); Department of Community Health and Family Medicine, University of Florida - Jacksonville (REJ).

Funding: None.

Conflict of interest: None.

Corresponding author: Peter J. Carek, MD, MS, Department of Community Health and Family Medicine, College of Medicine, University of Florida, PO Box 100237, Gainesville, FL 32610-0237 (E-mail: carekpj@musc.edu). procedures. ${ }^{5}$ In general, patients who are referred to specialists incur greater health care spending compared with those who remain within primary care, even after adjusting for health status. ${ }^{6}$ In addition, patients may not receive the care needed due to lack of referral completion, as about $17 \%$ of referred patients do not see the specialist to whom they are referred. ${ }^{7}$

The frequency of referrals made by primary care providers to a subspecialist physician varies widely. In earlier studies, the mean observed referral rates were $1.4 \%, 10.5 \%$, and $37 \%$, respectively. ${ }^{8-10}$ More recently, the combined referral rate of family medicine practices associated with residency programs varied from $7 \%$ to $31 \%$ with a median of $25 \% .{ }^{11} \mathrm{In}$ addition, concerns have been raised about an increase in overall referral rates, as the probability of receiving a referral during an ambulatory patient visit increased by $94 \%$ between 1999 and $2009 .^{12}$

While many issues influence referral rates, most factors are related to either the individual patient or 
physician. ${ }^{13}$ Patient characteristics affecting referral rates include presenting problem, patient expectations, and degree of morbidity. Physician characteristics include type of training, years of experience, experience in treating the condition affecting the patient, and certainty of diagnosis. Other individual physician factors that influence referral rates include practice style, technical orientation of care, fear of malpractice suits, and practice environment. Specialist characteristics such as perceived clinical skill, prior interactions, availability, and "returning" the patient to the referring physician also influence the variation in referral rates. Finally, the community, eg, urban versus rural, in which the provider practices often impacts the decision to refer. ${ }^{13}$

While playing a vital role in providing appropriate patient care, some referrals may be unnecessary. In 1 study, a group of primary care physicians believed that $33 \%$ of referrals were potentially avoidable. ${ }^{14}$ The physicians believed the referrals could have been avoided through training in simple procedures or communication with another generalist or specialist colleague.

Further understanding the referral rates and patterns by family physicians and practices will provide insight into opportunities to improve quality of care, reduce overall costs of care, and possibly enhance physician training, both graduate and continuing medical education, as to target commonly referred presenting complaints or diagnoses. While a frequent activity, few current studies have reviewed the volume and variations of referrals by practices associated with family physicians. Therefore, the aim of this study was to examine the current referral rates and patterns of family physician practices associated with a large academic health center. In addition to wide variation in rates and patient-specific factors, overall patient volume may have an impact on referral rates.

\section{Methods}

We conducted a retrospective chart review of referrals placed for patients seen in the family medicine clinics associated with a large academic institution between July 1, 2014 and June 30, 2018. This review included $1,310,420$ patient care visits to 30 practices that included 125 attending physicians (MD/DO), 25 family medicine residents, and 40 advanced practice providers (APPs) (advanced practice registered nurses/physician assistants). The unit study was the practice. The referrals of individual providers or provider groups (ie, attending physician, resident, or APP) were not able to be differentiated. No practice had more than 1 APP, and all referrals by a resident needed approval by an attending family physician.

For each practice site, basic demographic information was collected, including gender, race, ethnicity, age, marital status, and insurance status, on patients who received a referral and the specialty of the physician for whom the referral was sent. The data were summarized into monthly increments for analysis. For this study, a referral was any documented request by a physician or APP associated with our primary care department for a patient to be seen and evaluated by a specialist physician or APP either as a consult (onetime visit) or referral (ongoing care).

The primary outcome was the monthly referral rate, measured as the proportion of patient visits that resulted in a consult to a specialist outside of the referring family medicine clinic. In addition, the average length of the patient care encounter that produced the referral was obtained. Family medicine clinics were separated into 3 groups (high, medium, and low) based on their average monthly referral rates. The top third were designated as high referral clinics, the middle third as medium, and the bottom third as low.

Descriptive statics were used to provide demographic information. Binomial and $\gamma$ generalized linear mixed models (GLMMs) were fit to determine the associations between high/medium/low referral rate clinics and patient demographics. An adjusted GLMM was also fit to assess changes in referral rates over time. Each model included random effects for each clinic and fixed effects for clinic cluster (ie, high/medium/low referral clinics) and time.

\section{Results}

The referral rates, average encounter length that produced the referral, and patient demographics are presented in Table 1. The average monthly referral rate for all the clinics was $20.3 \%$, and the rate varied significantly between clinic groups (range: 0.4\%-67.1\%). Age and gender were similar across clinic groups, except a difference in gender was found between high and lower referring clinic 


\begin{tabular}{|c|c|c|c|c|c|c|c|}
\hline & \multirow[b]{2}{*}{ Overall } & \multirow[b]{2}{*}{ High } & \multirow[b]{2}{*}{ Medium } & \multirow[b]{2}{*}{ Low } & \multicolumn{3}{|c|}{$P$} \\
\hline & & & & & $\begin{array}{l}\text { High v } \\
\text { Medium }\end{array}$ & $\begin{array}{l}\text { Medium v } \\
\text { Low }\end{array}$ & $\begin{array}{l}\text { High v } \\
\text { Low }\end{array}$ \\
\hline Monthly referrals & 198 & 362 & 146 & 83 & & & \\
\hline Monthly visits & 973 & 1304 & 723 & 855 & & & \\
\hline Monthly referral rate & $20.3 \%$ & $27.8 \%$ & $20.2 \%$ & $9.9 \%$ & $<0.001$ & $<0.001$ & $<0.001$ \\
\hline $\begin{array}{l}\text { Avg encounter length } \\
\text { (minutes) }\end{array}$ & $64.9(23.1)$ & $69.3(22.9)$ & $64.0(25.6)$ & $61.0(19.6)$ & 0.927 & 0.967 & 0.881 \\
\hline \multicolumn{8}{|l|}{ Age of patients } \\
\hline 18 to 44 & $65(32.7 \%)$ & $120(33.2 \%)$ & $48(33.2 \%)$ & $26(31.6 \%)$ & 0.999 & 0.925 & 0.422 \\
\hline 45 to 64 & $91(45.7 \%)$ & $165(45.5 \%)$ & 65 (44.4\%) & $39(47.5 \%)$ & 0.906 & 0.824 & 0.641 \\
\hline $65+$ & $43(21.6 \%)$ & $77(21.3 \%)$ & $33(22.4 \%)$ & $17(20.9 \%)$ & 0.833 & 0.836 & 0.645 \\
\hline \multicolumn{8}{|l|}{ Gender } \\
\hline Female & $124(62.8 \%)$ & $231(63.9 \%)$ & $95(65.0 \%)$ & $49(59.4 \%)$ & 0.869 & 0.444 & $<0.001$ \\
\hline Male & $74(37.2 \%)$ & $131(36.1 \%)$ & $51(35.0 \%)$ & $34(40.6 \%)$ & & & \\
\hline \multicolumn{8}{|l|}{ Race/ethnicity } \\
\hline Non-Hispanic White & $117(59.3 \%)$ & $156(43.0 \%)$ & $84(57.2 \%)$ & $66(79.2 \%)$ & 0.004 & 0.001 & $<0.001$ \\
\hline Non-Hispanic Black & $58(29.5 \%)$ & $171(47.3 \%)$ & $37(25.2 \%)$ & $12(14.1 \%)$ & $<0.001$ & 0.078 & $<0.001$ \\
\hline Hispanic & $12(6.0 \%)$ & $21(5.8 \%)$ & $14(9.3 \%)$ & $2(2.9 \%)$ & 0.183 & 0.075 & 0.247 \\
\hline Other & $11(5.3 \%)$ & $14(3.9 \%)$ & $12(8.3 \%)$ & $3(3.8 \%)$ & 0.073 & 0.282 & 0.999 \\
\hline \multicolumn{8}{|l|}{ Marital status } \\
\hline Committed relationship & $86(43.6 \%)$ & $125(34.6 \%)$ & $65(44.3 \%)$ & $44(53.3 \%)$ & 0.029 & 0.272 & 0.022 \\
\hline $\begin{array}{l}\text { No committed } \\
\text { relationship }\end{array}$ & $112(56.4 \%)$ & $240(66.4 \%)$ & $81(55.7 \%)$ & $39(46.7 \%)$ & & & \\
\hline \multicolumn{8}{|l|}{ Payer type } \\
\hline Medicaid & $47(23.8 \%)$ & $118(32.6 \%)$ & 35 (23.6\%) & $12(14.3 \%)$ & 0.070 & 0.123 & $<0.001$ \\
\hline Medicare & $56(28.4 \%)$ & $110(30.5 \%)$ & 38 (25.9\%) & $24(28.7 \%)$ & 0.384 & 0.750 & 0.481 \\
\hline Charity & $2(0.9 \%)$ & $9(2.6 \%)$ & $<1(<0.1 \%)$ & $<1(<0.1 \%)$ & 0.332 & 0.999 & 0.674 \\
\hline Self-pay & $8(3.9 \%)$ & $12(3.4 \%)$ & $6(3.8 \%)$ & $4(4.4 \%)$ & 0.862 & 0.999 & 0.873 \\
\hline
\end{tabular}

groups, as the high referral clinicals disproportionately referred more female patients.

For non-Hispanic White patients, the referrals were significantly higher in the low referral clinics (79.2\%) compared with the high and medium clinics (43.0\% and $57.2 \%$, respectively). For non-Hispanic Black patients, the referrals were significantly higher in the high referral clinics $(47.3 \%$ compared with $25.2 \%$ and $14.1 \%$ ). Decreasing clinic referral rate was associated with a higher proportion of White patients who received referrals. The inverse was observed for Black patients. Relationship status was associated with clinic referral rates, as patients in committed relationships had lower referral rates, with the rates varying by clinic group. In terms of payor type, a difference was only noted in the referral rates for patients with Medicaid between high and low referral rate clinics.

Next, the referral rates were compared with the demographic characteristics of the total patient population (Table 2). For the overall clinic population as well as the high medical referral rate clinics, a disproportionate rate of referrals was noted for the lower aged groups (18 to 44 and 45 to 64 years of age) compared with the older aged group ( $\geq 65$ years of age).

The results of an adjusted regression analysis modeling the change in referral rate over time found the average referral rate across all clinics increased by $0.2 \%$ each month $(\mathrm{RR}=1.002$; $P<.001$ ) over the study time frame (Table 3 ). The evolution of the referral rate over time was not found to differ between high, medium, and low referral rate clinic groups $(\mathrm{RR}=1.000$; $P=.607)$.

The most common specialties for referral were consistent between the 3 clinic groups. Physical medicine and rehabilitation, gastroenterology, obgyn, cardiology, surgery, otolaryngology, neurology, and dermatology were each listed in the top 10 
Table 2. Average Monthly Demographic Characteristics of the Total Patient Population of Clinics by Clinic Group

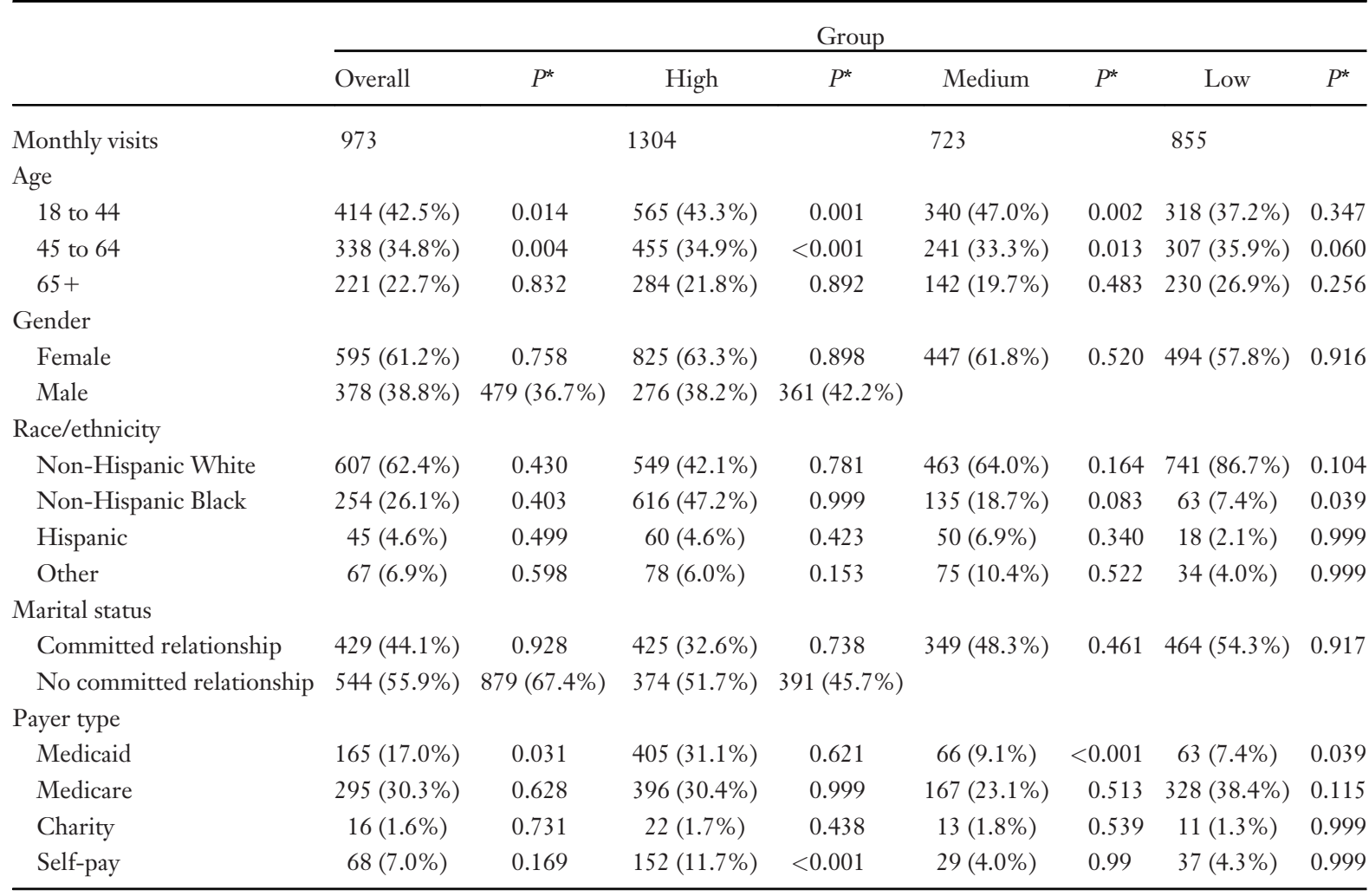

${ }^{*} P$ values represent comparisons between the proportion of patients referred versus the proportion of patients in the general clinic population.

Table 3. Adjusted Rate Ratios for the Change in Monthly Referral Rates over Time

\begin{tabular}{lcccc}
\hline & All Clinics* & $P$ & By Clinic Group & $P$ \\
\hline Monthly referral rate ratio & $1.002(1.001,1.003)$ & $<0.001$ & $1.000(0.999,1.001)$ & 0.607 \\
\hline
\end{tabular}

*Adjusted for encounter length, race, and insurance payer.

${ }^{\dagger}$ Model included a term for the interaction between time and high/medium/low referral clinic clusters.

most common specialties for referrals in each clinic group (Table 4).

\section{Discussion}

The referral rates among family medicine clinics associated with a single academic health center varied significantly, often by a factor of 2 to 3 , with a large range in values present (overall range: $0.4 \%-$ $67.1 \%$ and range of average rates between groups $9.9 \%-27.8 \%)$. The variations in referral rates were not surprising, as similar patterns have been noted in other family medicine departments. ${ }^{10,11}$ The magnitude of variations is significant and raises numerous questions regarding demographics and preferences of patients seen, acuity of patients being served, scope of practice of the physicians and advance practice providers, and other possible underlying factors.

To understand variations in referral rates, we examined several factors to determine if there was any correlation with the referral rate. While some factors were not correlated with referral rate, patient gender, race, and being in a committed relationship were noted to be associated with lower referral rates depending on the clinic overall referral rate. Of particular note, clinics with shorter encounter lengths were not associated with higher referral rates, suggesting referrals are not being made to hasten visits to meet certain expectations such as productivity 
Table 4. Top Ten Specialty Referrals by Clinic Group (Visits/Month)

\begin{tabular}{|c|c|c|c|}
\hline Overall & High Referral & Medium Referral & Low Referral \\
\hline 1. Gastroenterology (20.1) & 1. PMR (36.9) & 1. Gastroenterology (14.1) & 1. Gastroenterology (13.9) \\
\hline 2. PMR (15.6) & 2. Gastroenterology (34.6) & 2. PMR (11.1) & 2. Surgery (6.7) \\
\hline 3. Surgery (13.0) & 3. Ophthalmology (29.6) & 3. Surgery (10.4) & 3. Cardiology (6.1) \\
\hline 4. Ophthalmology (12.2) & 4. Surgery (24.1) & $\begin{array}{l}\text { 4. Obstetrics and gynecology } \\
(10.4)\end{array}$ & 4. Neurology (6.0) \\
\hline $\begin{array}{l}\text { 5. Obstetrics and gynecology } \\
(12.1)\end{array}$ & $\begin{array}{l}\text { 5. Obstetrics and gynecology } \\
(22.3)\end{array}$ & 5. Ophthalmology (7.6) & $\begin{array}{l}\text { 5. Obstetrics and gynecology } \\
(5.4)\end{array}$ \\
\hline 6. Neurology (9.7) & 6. Neurology (18.0) & 6. Otolaryngology (7.2) & 6. Otolaryngology (4.3) \\
\hline 7. Cardiology (9.6) & 7. Cardiology (17.7) & 7. Neurology (7.2) & 7. Dermatology (4.1) \\
\hline 8. Otolaryngology (7.8) & 8. Mental health (24.1) & 8. Cardiology (7.1) & 8. Urology (3.2) \\
\hline 9. Dermatology (7.7) & 9. Otolaryngology (14.8) & 9. Dermatology (7.0) & 9. PMR (2.9) \\
\hline 10. Mental health (6.6) & 10. Dermatology (14.5) & 10. Diabetes/endocrine (5.7) & 10. Diabetes/endocrine (2.7) \\
\hline
\end{tabular}

PMR, physical medicine and rehabilitation (includes rehabilitation, cardiac rehabilitation, and physical medicine and rehabilitation).

benchmarks or time constraints. The cause for these differences requires further study.

The referral rate across all clinics was found to increase over the time frame of the study. This observation is in-line with other studies that have also noted an increase in referral rates over the time periods studied. ${ }^{12}$ Although there was significant variation in referral rates that needs further investigation, the finding that the referred specialties are similar indicates that a need for additional training among family physicians in certain specialties is needed. Further characterization of referrals is needed to support a targeted approach to enhanced training among family physicians. In addition, the underlying factors associated with the increasing rates noted over time require further study.

Finally, referral rates impact cost and quality of care provided to individual patients and patient populations. The appropriate referral rate for family physicians that optimizes both cost and quality of care is yet to be determined. Further research in this area is both warranted and needed if family medicine wants to fulfill its Choosing Wisely commitment and follow the patient centered medical home model that "improves quality of care and medical outcomes, and helps reduce costs to the patient and health care system." ${ }^{15}$

This study was limited to patients seen and physicians and APPs practicing in a single institution in the southeastern United States. The referrals of individual providers or provider groups (ie, attending physician, resident, or APP) were not able to be differentiated. As such, the unit of study was the practice. The referral pattern of individual physicians could not be determined. In addition, whether the patients actually completed their referrals and were seen by the specialty physician is not known.

\section{Conclusion}

Referral rates among family physician practices differ widely and are associated with gender, race, and relationship status of patients. The length of an encounter with patients is not associated with referral rates. Finally, referrals to several specialties are common among family physicians. Although there are many factors affecting the referral rate, 1 area of opportunity can be seen in using commonly placed referrals to guide further training and education of residents and current faculty. Another area to explore is to see if multiple referrals are placed when patients expect multiple problems to be addressed in a time-limited clinic visit. Patient expectations may lead to an increase in referral rates seen in some clinics.

To see this article online, please go to: http://jabfm.org/content/ 34/6/1183.full.

\section{References}

1. Cleveland DR, Jindal KK, Hirsch DJ, Kiberd BA. Quality of prereferral care in patients with chronic renal insufficiency. Am J Kidney Dis 2002.

2. Boulware LE, Troll MU, Jaar BG, Myers DI, Powe NR. Identification and referral of patients with progressive CKD: a national study. Am J Kidney Dis 2006.

3. Kinchen KS, Sadler J, Fink N, et al. The timing of specialist evaluation in chronic kidney disease and mortality. Ann Intern Med 2002;137:479.

4. Wu AW, Young Y, Skinner EA, et al. Quality of care and outcomes of adults with asthma treated by specialists and generalists in managed care. Arch Intern Med 2001;161:2554-60.

5. Nutting PA, Franks P, Clancy CM. Referral and consultation in primary care: do we understand what we're doing? J Fam Pract 1992;35:21-3. 
6. Greenfield S, Nelson EC, Zubkoff $M$, et al. Variations in resource utilization among medical specialties and systems of care: results from the Medical Outcomes Study. JAMA 1992;267:1624.

7. Forrest CB, Shadmi E, Nutting PA, Starfield B. Specialty referral completion among primary care patients: results from the ASPN referral study. Ann Fam Med 2007;5:361-7.

8. Crump WJ, Massengill P. Outpatient consultations from a family practice residency program: nine years' experience. J Am Board Fam Pract 1998;1:164-6.

9. Tabenkin H, Oren B, Steinmetz D, Tamir A, Kitai E. Referrals of patients by family physicians to consultants: a survey of the Israeli Family Practice Research Network. Family Practice 1998;15:158-64.

10. Franks P, Zwanziger J, Mooney C, Sorbero M. Variations in primary care physician referral rates. Health Serv Res 1999;34:323-9.
11. Gwynne M, Page C, Reid A, Donahue K, Newton $W$. What's the right referral rate? Specialty referral patterns and curricula across 13 collaborative primary care residencies. Fam Med 2017;49:91-6.

12. Barnett ML, Song Z, Landon BE. Trends in physician referrals in the United States, 1999-2009. Arch Intern Med 2012;172:163.

13. Mehrotra A, Forrest CB, Lin CY. Dropping the baton: specialty referrals in the United States. Milbank Q 2011;89:39-68.

14. Donohoe MT, Kravitz RL, Wheeler DB, Chandra R, Chen A, Humphries N. Reasons for outpatient referrals from generalists to specialists. J Gen Intern Med 1999;14:281-6.

15. American Board of Internal Medicine. Choosing Wisely. Available from: https://www.choosingwisely. org/our-mission/specialty-society-partners/. Accessed April 27, 2021. 